\title{
Análise de satisfação e desempenho dos serviços e produtos ofertados em uma empresa do setor agrícola
}

\author{
GUSTAVO FELIPE SCHAEDLER* \\ SILVANA ANITA WALTER** \\ CINARA KOTTWITZ MANZANO BRENZAN*** \\ MÁRIO LUIZ SOARES****
}

* Administraçâo. Universidade Estadual do Oeste do Paraná, Marechal Cândido Rondon, Brasil. E-mail: gustavo.schaedler@outlook.com. ORCID: 0000-0002-3671-3219. Google Scholar: https://scholar.google.com/ citations?view_op=list_works\&hl=pt-BR\&authuser=2\&user=P7lQfhoAAAAJ.

** PhD em Administração. Universidade Estadual do Oeste do Paraná, Marechal Cândido Rondon, Brasil. E-mail: silvanaanita.walter@gmail.com. ORCID: 0000-0003-1684-5465. Google Scholar: https://scholar.google.com/ citations? user=t7ME1RAAAAAJ.

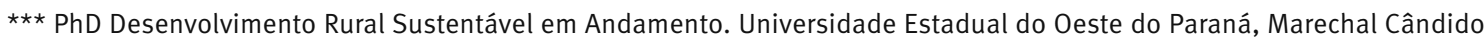
Rondon, Brasil. E-mail: cinaramanzano@yahoo.com.br. ORCID: 0000-0002-1684-2320. Google Scholar: https://scholar. google.com/citations?view_op=list_works\&hl=pt-BR\&user=SNu5n9EAAAAJ.

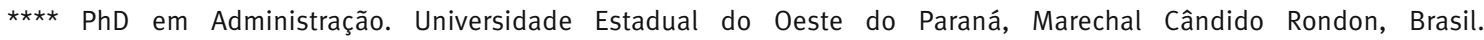
E-mail: mario.soares@unioeste.br. ORCID: 0000-0002-0561-8673. Google Scholar: https://scholar.google.com/ citations? authuser $=1$ \&user $=$ ppExq-kAAAJ. 


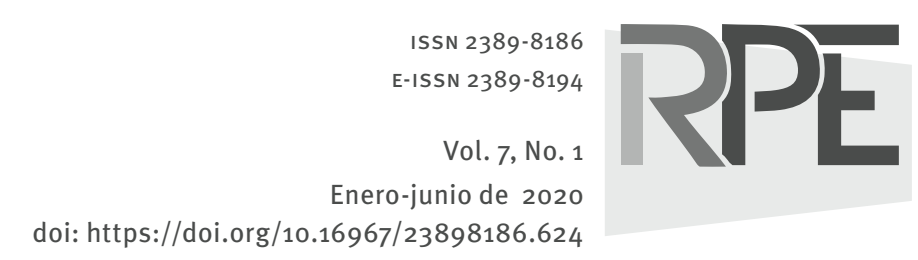

COMO CITAR ESTE ARTÍCULO How to cite this article:

Schaedler, G.F. et al. (2020). Análise de satisfação e desempenho dos serviços e produtos ofertados em uma empresa do setor agrícola. Revista Perspectiva Empresarial, 7(1), 25-45.

Recibido: 11 de noviembre de 2019

Aprobado: 22 de enero de 2020
RESUMO 0 presente artigo tem como objetivo a identificação de oportunidades de melhoria na satisfação de clientes de uma empresa do setor agrícola localizada no distrito de Novo Sarandi, Oeste do Paraná (Brasil), através do uso conjunto de dois métodos de análise, sendo eles: Modelo Kano e a Matriz de Importância X Desempenho; objetivando analisar o grau de satisfação e importância dos serviços prestados, permitindo uma melhor identificação dos atributos prioritários para melhoria. Para mensuração da satisfação utilizou-se uma pesquisa quantitativa descritiva, por meio de um questionário contendo 18 atributos, aplicado com 100 clientes fixos ou eventuais, que aceitaram respondê-lo. Constatou-se que a média de Satisfação Geral é mediana e que os clientes possuem nível Atrativos e Unidimensionais em relação a melhoria de produtos. Os atributos atrativos apresentam maior impacto na satisfação se melhorados do que insatisfação se piorados, sendo: aparência dos funcionários; aparência externa e aparência interna da empresa. Destaca-se pela análise dos dados, que a empresa atende as expectativas dos consumidores em alguns atributos, sendo que, dos 18 atributos avaliados, é necessário oportunizar a melhoria com mais atenção em cinco deles: agilidade no recebimento de produtos; preço dos produtos; descontos em relação aos produtos no pagamento a vista; disponibilidade de estoque e prontidão no atendimento; considerados atributos de alta importância ao cliente, mas com baixo nível de desempenho.

PALAVRAS-CHAVE satisfação de cliente, Matriz de Importância X Desempenho, modelo Kano.

\section{Análisis de satisfacción y desempeño de servicios y productos ofrecidos en una empresa del sector agrícola}

RESUMEN Este artículo tiene como objetivo identificar oportunidades de mejora en la satisfacción del cliente de una empresa del sector agrícola ubicada en el distrito de Novo Sarandi, Oeste del Estado de Paraná (Brasil), mediante el uso conjunto de dos métodos de análisis: el modelo Kano y la matriz de importancia $X$ desempeño; esto con el objetivo de analizar el grado de satisfacción e importancia de los servicios prestados, permitiendo una mayor identificación de los atributos prioritarios para mejora. Para medir la satisfacción se utilizó una encuesta cuantitativa descriptiva, con un cuestionario de 18 atributos, aplicada a 100 clientes fijos u ocasionales que aceptaron responderla. Se encontró que la satisfacción general es promedio y que los clientes tienen niveles atractivos y unidimensionales en relación con la mejora del producto. Los atributos atractivos tienen un mayor impacto en la satisfacción si se mejora: la apariencia de los empleados; la apariencia externa y la apariencia interna de la empresa. Destaca por el análisis de los datos que la empresa cumple con las expectativas de los consumidores en algunos atributos, siendo necesario brindar una mejora en cinco de ellos: agilidad en la recepción de productos; precio de los productos; descuentos en relación con productos de pago en efectivo; disponibilidad de stock y servicio rápido; todos ellos considerados atributos de gran importancia para el cliente, pero con un bajo nivel de rendimiento. 
PALABRAS CLAVE satisfacción del cliente, matriz de importancia X desempeño, modelo Kano.

\section{Satisfaction and performance analysis of services and products offered by a company from the agricultural sector}

ABSTRACT This paper aims at identifying improvement opportunities in customer satisfaction of a company from the agricultural sector located in the Novo Sarandi district, West of the Paraná State (Brazil). This was done through the joint use of two analysis methods: The Kano model and Mandatory Quality and the importance $X$ performance matrix; with the purpose of analyzing the degree of satisfaction and importance of the services rendered, allowing greater identification of priority attributes to be improved. To measure satisfaction, a quantitative descriptive survey was used, with a questionnaire of 18 attributes, applied to 100 regular or occasional customers that accepted to respond it. It was found that general satisfaction is average and that customers have attractive and unidimensional levels in relation to product improvement. Attractive attributes have a greater impact on satisfaction if these are improved: employees' appearance and the company's internal and external appearance. The data analysis shows that the company meets consumers' expectations in some attributes, 5 of which need improvement: agility in product reception; product price; discounts on cash purchases; stock availability; and quick service; all of which are considered attributes of great importance for the customer, but have a low performance level.

KEY WORDS customer satisfaction, importance x performance matrix, Kano model for Attractive and Mandatory Quality. 


\section{Introdução}

Quando empresas se mantêm líderes por muitos anos e em diferentes setores, uma de suas habilidades gerenciais é a de medir seu próprio desempenho buscando sempre melhores resultados. Empresas que não buscam um melhoramento de seu desempenho são mais vulneráveis às mudanças no ambiente externo. Organizações que visam para indicadores financeiros geralmente não refletem a verdadeira realidade e nem garantem a sustentabilidade.

Para compreender melhor a gestão de sua empresa os reflexos e impactos causados aos seus clientes a pesquisa de satisfação tende a ser umas das melhores ferramentas. Pois, através desta pode-se entender como seu cliente pensa, em relação ao desempenho, qualidade dos produtos e dos serviços prestados, buscando melhorar no que for possível e aproveitar o que já é visto como bom (Milan, 2005).

Estudos evidenciam que a satisfação dos clientes pode ocasionar o aumento da lealdade, como consequência pode-se assegurar receitas a longo prazo, reduzir custos de transações futuras, diminuir a elasticidade de preços e diminuir a probabilidade de trocarem de empresa caso haja uma queda na qualidade (Anderson, Fornell and Rust, 1997; Anderson, 1998; Anderson and Fornell, 1999). Para que uma organização se mantenha eficiente, o controle é fundamental. Um sistema de medição de desempenho é uma forma eficiente de controlar e auxiliar na tomada de decisões no nível estratégico ou operacional (Salazar, 2001).

Sendo assim, esta pesquisa aborda aspectos de satisfação e não satisfação dos clientes em uma empresa agrícola de Toledo, região oeste do Paraná, tendo como pergunta principal: Qual a importância e o grau de satisfação dos clientes em relação aos serviços e produtos ofertados pela empresa?

Para identificar, 18 atributos foram selecionados para análise. Utilizou-se a Matriz de Importância X Desempenho para análise das oportunidades de melhoria pois assume-se que a satisfação de um cliente está relacionada com o desempenho dos diversos atributos de um serviço ou produto. Utilizou-se também o modelo Kano como uma alternativa de identificar o efeito do desempenho dos atributos na satisfação dos clientes por não haver linearidade entre desempenho e satisfação (Pereira, Tontini e Silveira, 2005).

Esta pesquisa tem como seu objetivo analisar a satisfação dos clientes de uma empresa do setor agrícola, em relação aos serviços e produtos ofertados, foram utilizados dois modelos de mensuração, aplicados a empresa Agrícola Horizonte, localizada em Novo Sarandi, Toledo, Brasil.

Para tanto se seguiram as etapas: (i) identificar a satisfação atual dos clientes com relação a cada atributo; (ii) averiguar a importância de cada atributo para seus colaboradores; (iii) avaliar a satisfação geral com a empresa; (iv) inspecionar a satisfação do cliente se um atributo melhorar ou piorar; (v) evidenciar pontos fortes, fracos e sugestões de melhoria.

0 artigo encontra se estruturado em cinco seções. A primeira trata do tema, problema, objetivos e a justificativa para a presente pesquisa. Na segunda apresenta-se a base teóricoempírica sobre satisfação de clientes, Matriz de Importância X Desempenho, modelo Kano e estudos empíricos. Na terceira seção, descrevemse os procedimentos metodológicos empregados para o desenvolvimento do estudo de caso. $\mathrm{Na}$ quarta, tem-se a análise dos dados obtidos, abrangendo a descrição do caso e a análise de satisfação ou insatisfação dos clientes da empresa. $\mathrm{Na}$ quinta seção, destacam-se as considerações finais, incluindo a conclusão e limitações da pesquisa, assim como sugestões para trabalhos futuros.

\section{Referencial teórico}

\section{Satisfação de clientes}

0 comprometimento absoluto com o cliente é o diferencial que a empresa precisa buscar no mercado atual, por isso o conceito de satisfação do cliente está presente nas 
organizações, pois a satisfação é a base de qualquer sucesso consistente, de médio ou longo prazo (Candeloro e Almeida, 2002).

Satisfação de modo geral, é um sentimento ou reação sobre o uso e consumo de um determinado produto ou serviço ofertado por uma empresa (Solomon, 1999). É a função entre o desempenho e a expectativa, se o desempenho for bom, o cliente ficará satisfeito, se o desempenho não for bom o cliente ficará insatisfeito. Se o desempenho exceder as expectativas do consumidor, ele ficará encantado, companhias voltadas para o marketing desviam-se de seu caminho para manter os clientes satisfeitos. Pois isso faz com que eles repitam suas compras e falem para outros sobre as boas experiências com os produtos ou serviços (Kotler, 2000; Kotler e Armstrong, 1999). A satisfação dos clientes pode afetar a reputação de uma forma positiva ou negativa, devido aos avanços na comunicação digital e pessoal, sendo que clientes não satisfeitos podem causar um grande impacto na rentabilidade de uma empresa (Babin and Harris, 2012).

Marketing está relacionado a obter mercadosalvos, manter e multiplicar clientes por meio da criação, e de uma valorização superior para os clientes. Uma administração de marketing bemsucedida exige estratégias e planos de marketing, uma conexão com os clientes, construção de marcas fortes, ofertas de mercado, de captura de oportunidades de marketing, e uma relação de longo prazo entre cliente e fornecedor (Kotler e Keller, 2006).

A necessidade de compra do consumidor consiste em várias etapas: (i) reconhecer a necessidade de compra seja por uma vontade pessoal ou por algum problema enfrentado; (ii) busca de informações sobre os produtos ou serviços, os quais indicam se os mesmos têm a possibilidade de atender a sua necessidade pessoal; (iii) uma decisão de compra levando em conta todos os produtos que o mercado pode oferecer; (iv) pós compra, fator muito importante para o marketing da empresa, pois é onde o cliente vai medir a sua satisfação perante a compra do produto (Kotler e Armstrong, 1999).

0 custo para uma empresa conseguir novos clientes é significativamente maior do que os custos para ela manter os já conquistados, isso faz com que ela procure satisfazer os clientes que já possui (Bateson e Hoffman, 2001). Apenas satisfazer os clientes não é suficiente, as empresas precisam mantê-los satisfeitos para que tenham um relacionamento de longo prazo, e que se tornem menos propensos a trocar de fornecedor ou marca (Zeelenberg and Pieters, 2004).

Para se ter uma vantagem competitiva em relação aos concorrentes, a satisfação dos clientes é um item indispensável para uma organização. Para analisar a satisfação dos clientes em relação às suas expectativas sobre os produtos e serviços de uma empresa, pode-se utilizar métodos lineares e métodos não lineares. No presente estudo foram utilizadas duas metodologias: a Matriz de Importância X Desempenho (método linear) e o modelo Kano (método não linear) respectivamente.

\section{Matriz de Importância X Desempenho}

A análise de importância e desempenho, proposta por Martilla e James (1977), permite a avaliação de atributos dos produtos e serviços ofertados pela empresa, para que haja uma melhoria se necessário, levando em conta a alta competitividade do mercado. Permite aos gestores a visualização da relação entre a intensidade da importância dos atributos relacionados a um problema e a intensidade do desempenho da organização. Os dados provenientes da pesquisa de satisfação dos clientes são utilizados para a construção de uma matriz bidimensional, na qual a importância é mostrada pelo eixo Y e o desempenho do atributo pelo eixo X (figura 1).

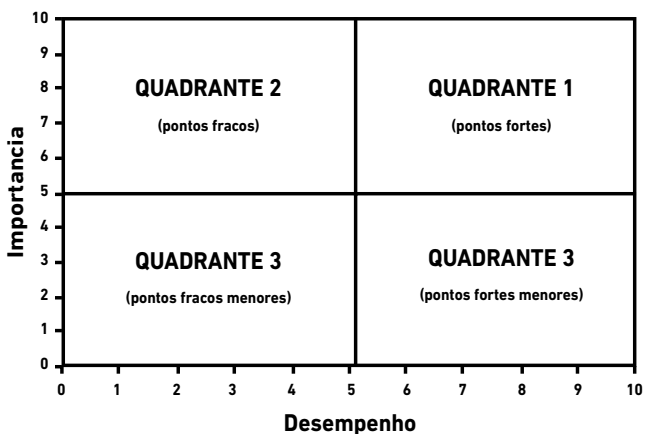

Figura 1. Matriz de Importância X Desempenho. Fonte: Tontini et al. (2004, p. 4). 
A matriz é dividida em quatro quadrantes: o atributo no Quadrante I terá alta importância com alto desempenho, representando uma vantagem competitiva, assim sendo a empresa deve manter o trabalho realizado referente ao produto ou serviço. 0 atributo que se encontra no Quadrante II terá alta importância e baixo desempenho, visando que medidas devem ser tomadas rapidamente pela empresa para melhorar a satisfação geral, minimizando a possível perda de clientes. No Quadrante III encontramse os atributos de baixa importância e baixo desempenho, não sendo necessário concentrar esforços para melhorá-los, pois não são relevantes para um descontentamento do consumidor. E os atributos do Quadrante IV possuem um alto desempenho, mas com baixa importância, o que poderia acarretar o desperdício de recursos pelas empresas, sendo que esses recursos poderiam ser utilizados em atributos de alta importância e baixo desempenho.

A análise da matriz de importância e desempenho sugere que a relação entre o desempenho e a satisfação é linear, isso pode gerar decisões equivocadas sobre quais produtos ou serviços devem ser melhorados ou acrescentados nas organizações (Tontini and Silveira, 2005).

Entretanto, o desempenho de um atributo e a satisfação por ele gerada é mais complexa, não apenas não linear, mas também assimétrica, sendo que a importância pode variar conforme o desempenho, trazendo um maior grau de análise das oportunidades de melhoria (Matzler et al., 2004). Assim, o modelo Kano traz uma perspectiva diferente para a análise de possíveis melhorias por levar em consideração a relação assimétrica entre desempenho e satisfação.

\section{Modelo Kano}

Modelo Kano apresenta a maneira pela qual um atributo de produto afeta a satisfação do cliente. Esse modelo exibe a relação entre o comprimento físico de um atributo de qualidade em um produto e a satisfação percebida desse atributo. As relações não são iguais para todos os atributos de qualidade e também mudam com o tempo (Kano et al., 1984).

De acordo com Tontini (2003), o gráfico abaixo (figura 2) apresenta o desenvolvimento de uma metodologia modificada para identificação dos atributos dentro do modelo Kano, permitindo a determinação do grau de satisfação ou não satisfação que a existência ou a inexistência de um atributo pode trazer aos consumidores.

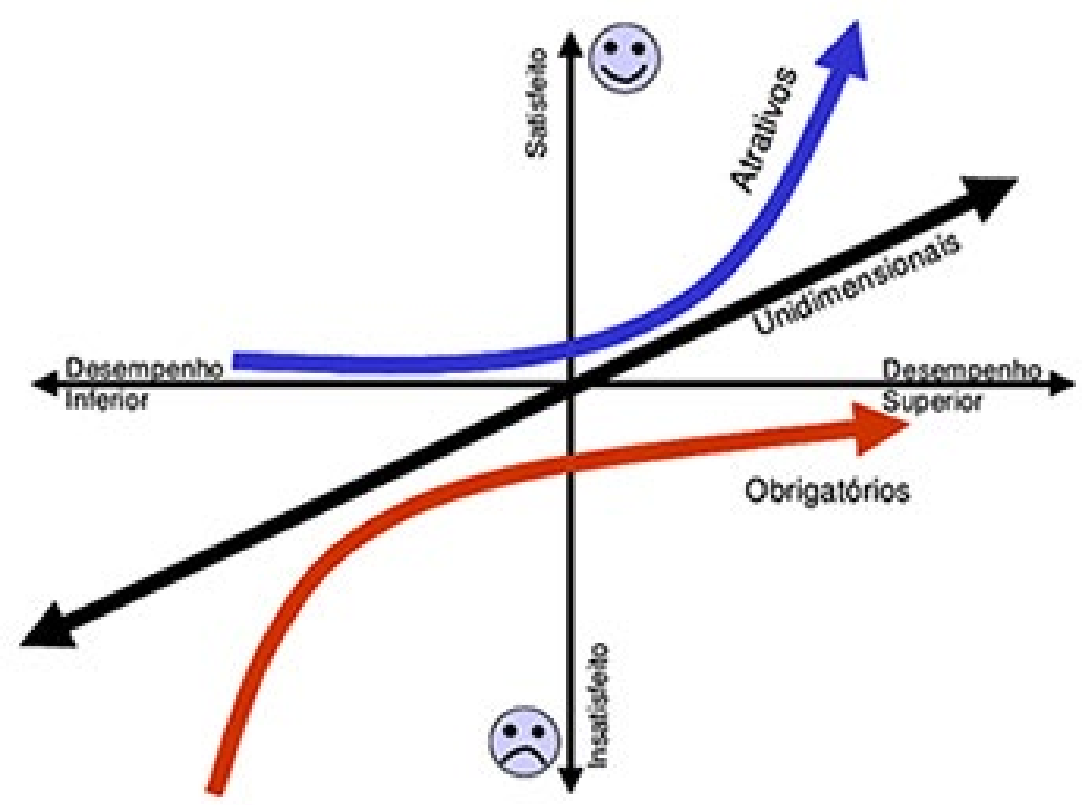

Figura 2. Modelo Kano. Fonte: Tontini et al. (2004, p. 7). 
Complementando a ideia, Tontini e Silveira (2007) propõe que o modelo Kano é a ligação entre desempenho e satisfação não linear, classificando os atributos como obrigatórios, unidimensionais e atrativos.

Atributos obrigatórios: preenchem as funções básicas de um produto. Se esses atributos não estiverem presentes ou se o desempenho dos mesmos for baixo, os clientes ficarão extremamente insatisfeitos. Por outro lado, mesmo que esses atributos estejam presentes ou tenham bom desempenho, não trazem satisfação. Usando o exemplo de um estabelecimento de alimentação, se o ambiente estiver com a limpeza correta, o cliente não se sentirá mais satisfeito, mas no caso da limpeza deixar a desejar possivelmente não retornará ao estabelecimento.

Atributos unidimensionais: a satisfação é proporcional ao nível de desempenho, quanto maior o nível de desempenho, maior será a satisfação do cliente e vice-versa. Como exemplo, podemos usar um automóvel que terá um gasto de combustível por quilômetro rodado, se essa quilometragem for maior por litro de combustível, mais satisfeito o consumidor ficará já se a quilometragem for baixa por litro de combustível o consumidor ficará insatisfeito com o resultado.

Atributos atrativos: são essenciais para a satisfação do cliente. 0 atendimento desses atributos traz uma satisfação mais que proporcional. Entretanto, não trarão insatisfação caso não forem atendidos. Por exemplo, se um restaurante oferecer uma 'lembrança' após um jantar, os clientes ficarão satisfeitos, mas se caso o restaurante não oferece, não trará insatisfação.

Atender as expectativas dos clientes foi resultante para o desenvolvimento do modelo Kano, não levando apenas em consideração o produto, mas também o desempenho que tal produto teria, levando a satisfação de seu consumidor, assim, o modelo foi idealizado na qualidade do ponto de vista do uso do produto e não diretamente ao produto (Kano, 1991).

\section{Estudos empíricos sobre o tema}

Realizando pesquisa para a identificação de oportunidades de melhoria em laboratórios fotográficos através da integração da Matriz de Importância X Desempenho com o modelo Kano, avaliaram a posição competitiva e oportunidades de melhoria para uma loja situada na cidade de Blumenau, Santa Catarina, comparando-a com outras lojas de fotografia das regiões de Criciúma, Içara e Blumenau, igualmente em Santa Catarina. Foram aplicados 300 questionários distribuídos proporcionalmente ao número da população de cada cidade (Tontini and Silveira, 2005).

A integração dos dois métodos permitiu superar as limitações apresentadas pela Matriz de Importância X Desempenho. Concluiu-se que a loja estudada deve continuar com o bom atendimento e observar que um layout agradável com boa iluminação pode ser bastante atrativo; a ampliação de brinde deve ser mantida ou melhorada; o tempo de revelação deve acompanhar as tendências do mercado; a variedade de produtos e serviços pode ser mantida ou ser incrementada; a loja deve rever sua posição como marca exclusiva, pois este constitui um atributo que traz insatisfação ao consumidor.

\section{Metodologia}

Para a elaboração desta pesquisa utilizou-se o método quantitativo, e o instrumento utilizado para coleta de dados foi um questionário baseado no modelo Kano proposto por Tontini (2003), explorando a satisfação dos clientes da Agrícola Horizonte, com os atributos existentes na relação a produtos e serviços ofertados pela empresa. Para o cálculo do tamanho da amostra dessa etapa, foi utilizada a equação citada por Mattar (1996), para uma população finita:

$$
n=\frac{N Z{ }^{2} P Q}{\left(E^{2} N+Z^{2} P Q\right)}
$$


Onde: $\mathrm{n}$ : tamanho da amostra; N: tamanho da população; P: proporção de ocorrência da variável em estudo na população $(0,5)$; Q: proporção de não ocorrência da variável em estudo na população $(\mathrm{Q}=1$ - $\mathrm{P})$; E: erro máximo admitido $(0,05)$; Z: valor padrão determinado pelo nível de confiança. Para o nível de confiança de $95 \%$, Z= 2 .
0 resultado do cálculo do tamanho mínimo da amostra foi de 100 questionários, a margem de erro conforme este cálculo é de $5 \%$. A Agrícola Horizonte está relacionada ao setor agrícola e possui 150 clientes cadastrados, foram analisados 100 questionários, os quais representam um percentual de 65 a $67 \%$ dos clientes da empresa.

$\mathrm{n}=150 \cdot 2^{2} \cdot(0,5 \cdot 1-0,5) /\left(0,05^{2} \cdot 150+2^{2}\right) \cdot(0,5$

$.1-0,5)$

$\mathrm{n}=600 \cdot 0,25 / 0,0025 \cdot 150+4 \cdot 0,25$

$\mathrm{n}=1500 / 0,5+1$

$\mathrm{n}=100$

Tabela 1. Atributos utilizados para pesquisar a satisfação dos clientes

\begin{tabular}{ll}
\hline & Atributos \\
\hline Apresentação dos funcionários & Agilidade na entrega dos produtos \\
\hline Gentileza no atendimento dos funcionários & Agilidade no recebimento dos produtos \\
\hline Prontidão no atendimento dos funcionários & Explicação sobre o uso/ação dos produtos \\
\hline Organização da empresa & Atendimento pós venda \\
\hline Limpeza e higiene do escritório e barracões & Cumprimento com o que foi combinado \\
\hline Aparência externa da empresa & Descontos em relação aos produtos no pagamento a vista \\
\hline Aparência interna da empresa & Condições de pagamento \\
\hline Qualidade dos produtos & Preço dos produtos \\
\hline Diversidade dos produtos & - \\
\hline Disponibilidade de estoque & -
\end{tabular}

Fonte: elaboração própria pelos autores.

A empresa Agrícola Horizonte de Novo Sarandi, possui 150 clientes cadastrados, sendo analisados 100 questionários, aplicados a uma população diversificada, no que diz respeito à faixa etária, estado civil e ao sexo dos entrevistados.

O questionário foi composto por quatro etapas. Na primeira parte mediu-se a satisfação atual dos clientes em relação a cada atributo com a empresa, comparando o grau de satisfação existente em relação ao nível de desempenho atual encontrado nos atributos. A avaliação ocorreu numa escala variando de extremamente insatisfeito $(-5)$ a extremamente satisfeito $(+5)$, na qual o cliente indicou a sua satisfação ou não satisfação em relação a cada atributo apresentado no questionário, conforme mostra o tabela 2 .

Tabela 2. Questões para identificação dos atributos pelo modelo Kano

\begin{tabular}{llllllllllll}
\hline $\begin{array}{c}\text { Aponte a sua satisfação atual em } \\
\text { relação a cada atributo }\end{array}$ & \multicolumn{4}{c}{ Extremamente insatisfeito } & \multicolumn{3}{c}{$\begin{array}{c}\text { Neutro ou } \\
\text { indiferente }\end{array}$} & \multicolumn{2}{c}{ Extremamente satisfeito } \\
\hline Apresentação dos funcionários & -5 & -4 & -3 & -2 & -1 & 0 & 1 & 2 & 3 & 4 \\
\hline Gentileza no atendimento & -5 & -4 & -3 & -2 & -1 & 0 & 1 & 2 & 3 & 4 \\
\hline
\end{tabular}

Fonte: elaboração própria pelos autores. 
$\mathrm{Na}$ segunda parte, verificou-se o grau de importância que cada atributo tem para cada cliente, sendo atribuídas notas numa escala de importância de: Sem importância (1) a Extremamente importante (5), conforme apresenta o tabela 3 .

Tabela 3. Questões para identificação do grau de importância dos atributos pela Matriz de Importância X Desempenho

\begin{tabular}{|c|c|c|c|c|c|}
\hline $\begin{array}{l}\text { Indique o grau de } \\
\text { importância que } \\
\text { cada atributo tem } \\
\text { para você }\end{array}$ & Sem importância & $\begin{array}{c}\text { Alguma } \\
\text { importância }\end{array}$ & Importante & $\begin{array}{c}\text { Muito } \\
\text { importante }\end{array}$ & $\begin{array}{l}\text { Extremamente } \\
\text { importante }\end{array}$ \\
\hline $\begin{array}{l}\text { Apresentação dos } \\
\text { funcionários }\end{array}$ & 1 & 2 & 3 & 4 & 5 \\
\hline $\begin{array}{l}\text { Gentileza no } \\
\text { atendimento }\end{array}$ & 1 & 2 & 3 & 4 & 5 \\
\hline
\end{tabular}

Fonte: elaboração própria pelos autores.

No terceiro momento avaliou-se a satisfação geral atual dos clientes com a empresa, variando a escala de extremamente insatisfeito $(-5)$ a extremamente satisfeito $(+5)$, conforme demonstra o tabela 4.

Tabela 4. Avaliação do grau de satisfação geral com o supermercado

\begin{tabular}{|c|c|c|c|c|c|c|c|c|c|c|c|}
\hline \multirow{2}{*}{$\begin{array}{l}\text { Avalie seu grau de satisfação ou } \\
\text { insatisfação atual geral com a } \\
\text { agrícola }\end{array}$} & \multicolumn{5}{|c|}{ Extremamente insatisfeito } & \multicolumn{3}{|c|}{$\begin{array}{l}\text { Neutro ou } \\
\text { indiferente }\end{array}$} & \multicolumn{3}{|c|}{ Extremamente satisfeito } \\
\hline & -5 & -4 & -3 & -2 & -1 & 0 & 1 & 2 & 3 & 4 & 5 \\
\hline
\end{tabular}

Fonte: elaboração própria pelos autores.

$\mathrm{Na}$ quarta parte do questionário foram realizadas duas perguntas sobre cada um dos atributos, descrevendo respectivamente uma situação de aumento e outra de diminuição no desempenho de cada um deles, avaliando através de uma escala de -5 (extremamente insatisfeito) a +5 (extremamente satisfeito), como mostra o tabela 5 .

Tabela 5. Avaliação do grau de satisfação geral com o supermercado

\begin{tabular}{|c|c|c|c|c|c|c|c|c|c|c|c|}
\hline $\begin{array}{l}\text { Indique como você se sentiria em } \\
\text { relação a agrícola }\end{array}$ & \multicolumn{5}{|c|}{ Extremamente insatisfeito } & \multicolumn{3}{|c|}{$\begin{array}{l}\text { Neutro ou } \\
\text { indiferente }\end{array}$} & \multicolumn{3}{|c|}{ Extremamente satisfeito } \\
\hline $\begin{array}{l}\text { 1. Se a apresentação dos } \\
\text { funcionários for melhor que é } \\
\text { atualmente }\end{array}$ & -5 & -4 & -3 & -2 & -1 & 0 & 1 & 2 & 3 & 4 & 5 \\
\hline $\begin{array}{l}\text { 1.1 Se a apresentação dos } \\
\text { funcionários for pior que é } \\
\text { atualmente }\end{array}$ & -5 & -4 & -3 & -2 & -1 & 0 & 1 & 2 & 3 & 4 & 5 \\
\hline
\end{tabular}


$\mathrm{Na}$ última parte, pesquisaram-se pontos fortes, fracos e sugestões de melhoria por meio de perguntas abertas, e dados pessoais dos respondentes, como idade, sexo, estado civil, através de perguntas fechadas e objetivas.

A análise foi feita por meio de correlação estatística usando o SPSS versão 22.0. Também foram gerados gráficos no Excel com a lógica não linear do modelo Kano, bem como gráfico com a matriz de Importância X Desempenho.

\section{Resultados}

\begin{abstract}
Esta seção apresenta os dados divididos em cinco blocos: inicialmente, o perfil dos respondentes; depois, a análise conjunta da Matriz de Importância X Desempenho e modelo Kano além de análises estatísticas multivariadas, a análise de correlação no SPSS e na última etapa, os pontos fortes, fracos e sugestões de melhorias.
\end{abstract}

\section{Perfil dos respondentes}

Para iniciar as análises foram caracterizados os respondentes. Três questões fechadas relacionadas ao perfil dos clientes, conforme a tabela 6:

Tabela 6. Sexo dos respondentes

\begin{tabular}{lll}
\hline Quantidade & Quantidade & Percentual \\
\hline Feminino & 11 & $11 \%$ \\
\hline Masculino & 89 & $89 \%$ \\
\hline Total & 100 & $100 \%$ \\
\hline
\end{tabular}

Fonte: elaboração própria pelos autores.
Quanto ao gênero, percebe-se que a maioria dos respondentes é do sexo masculino, correspondendo a $89 \%$ do total, sendo $11 \%$ o percentual de respondentes do sexo feminino.

Em relação à faixa etária, os resultados sobre a idade dos participantes são apresentados na tabela 7: 
Tabela 7. Faixa etária dos respondentes

\begin{tabular}{|c|c|c|c|c|c|}
\hline Faixa etária & Quantidade & Percentual & Faixa Etária & Quantidade & Percentual \\
\hline o a 18 anos & 1 & $1 \%$ & 51 a 60 anos & 29 & $29 \%$ \\
\hline 19 a 25 anos & 7 & $7 \%$ & 61 a 70 anos & 9 & $9 \%$ \\
\hline 26 a 30 anos & 5 & $5 \%$ & 71 a 80 anos & 2 & $2 \%$ \\
\hline 31 a 40 anos & 19 & $19 \%$ & 80 a 100 anos & 0 & $0 \%$ \\
\hline \multirow[t]{2}{*}{41 a 50 anos } & 28 & $28 \%$ & - & - & - \\
\hline & & & Total & 100 & $100,00 \%$ \\
\hline
\end{tabular}

Fonte: elaboração própria pelos autores.

Observa-se, por meio da tabela 7 , que há predominância de um público adulto. A maior parte dos respondentes está na faixa etária de 41 a 60 anos, correspondendo a $57 \%$ do total.

O estado civil dos clientes da empresa é apresentado na tabela 8:

Tabela 8. Estado civil

\begin{tabular}{lcc}
\hline Estado civil & Quantidade & Percentual \\
\hline Casado(a) & 67 & $67 \%$ \\
\hline Solteiro(a) & 18 & $18 \%$ \\
\hline Viúvo(a) & 2 & $2 \%$ \\
\hline Amasiado(a) & 8 & $8 \%$ \\
\hline Divorciado(a) & 5 & $5 \%$ \\
\hline Total & 100 & $100 \%$ \\
\hline
\end{tabular}

Fonte: elaboração própria pelos autores.

Nota-se, a partir da tabela 8, que a maior parte dos respondentes é casado(a), representando 67 $\%$ do total.

\section{Satisfação geral}

Em relação à satisfação geral, os resultados são apresentados na tabela 9:

Tabela 9. Satisfação geral

\begin{tabular}{lc}
\hline Satisfação Geral & \\
\hline Média & 2,95 \\
\hline Percentual de satisfação & $81,36 \%$ \\
\hline
\end{tabular}

Fonte: elaboração própria pelos autores.
Baseando-se nos dados da tabela 9, percebe-se que as médias se apresentaram de forma positiva, sendo que a satisfação geral atinge percentual de satisfação igual à $81,36 \%$ de aprovação. Isto representa um nível de percepção de qualidade e satisfação medianos em relação aos produtos e serviços prestados pela loja. Por meio da análise dos dados, é possível observar que a empresa atende às expectativas e desejos dos clientes de forma geral.

\section{Discussões de análise comparativa: Matriz de Importância X Desempenho e modelo Kano}

Os resultados da aplicação dos questionários foram processados procurando-se identificar a classificação dos atributos segundo o modelo Kano e as oportunidades de melhoria pela Matriz de Importância X Desempenho.

\section{Análise pela Matriz de Importância X Desempenho}

A figura 3 mostra a Matriz de Importância X Desempenho para os atributos estudados. Um ponto chave na análise por essa matriz é a posição das linhas divisórias dos quadrantes. 


\section{Importância $x$ desempenho}

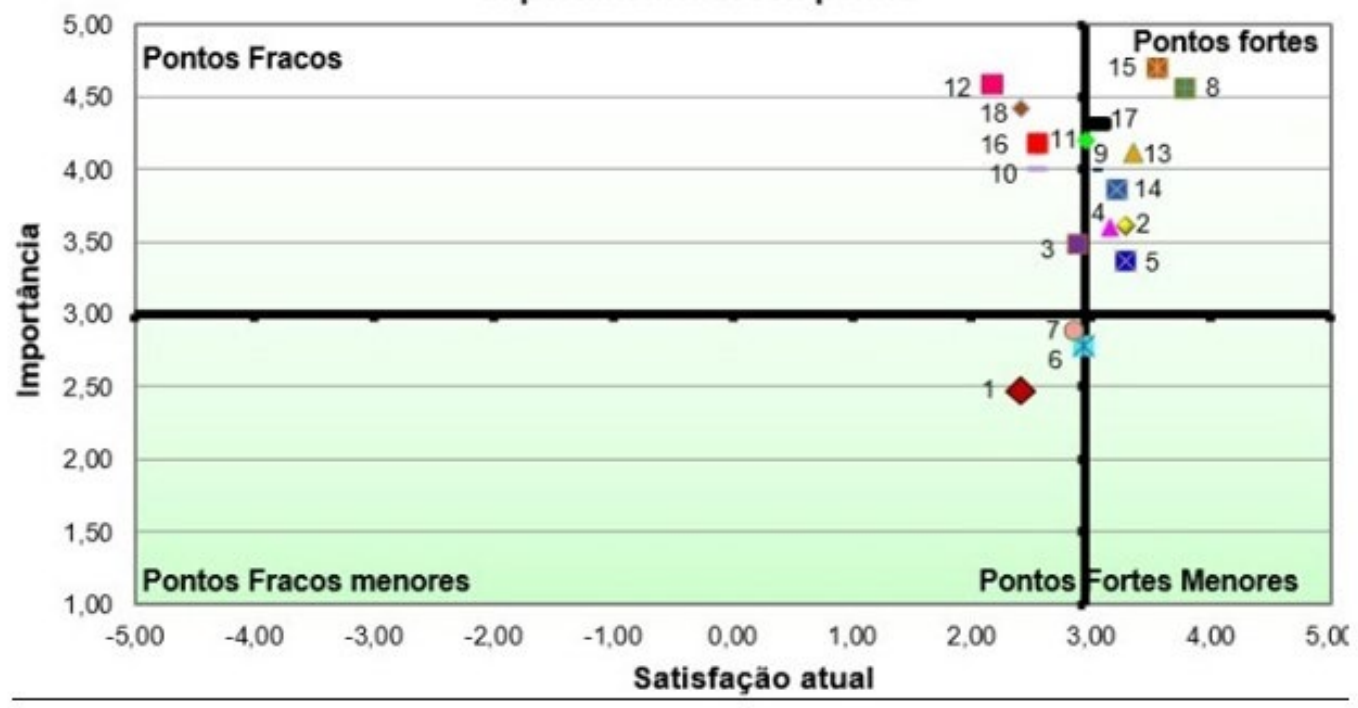

Figura 3. Matriz de Importância X Desempenho para atributos pesquisados. Fonte: elaboração própria pelos autores.

A figura 3 pode ser visualizada de forma ampliada (zoom), por meio da figura 4 .

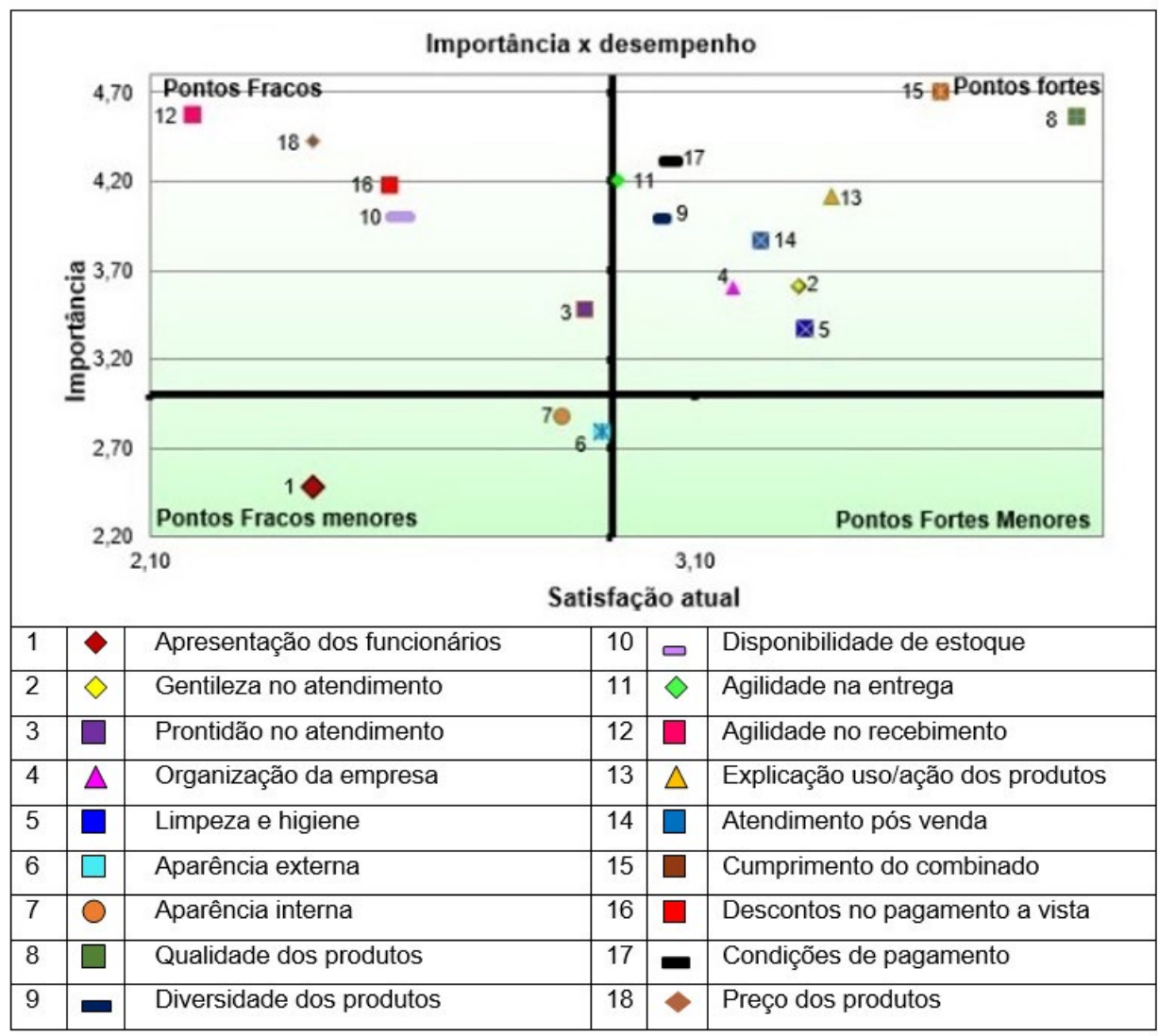

Figura 4. Ampliação do gráfico de dispersão do índice de satisfação. Fonte: elaboração própria pelos autores. 
Os quadrantes encontram-se divididos por uma linha estabelecida em importância (3), e outra de satisfação atual, estabelecida pela média de satisfação geral atual em relação aos atributos $(2,95)$.

No Quadrante I, da figura 4 observamse dez atributos, pontos fortes da pesquisa: qualidade dos produtos (8); cumprimento dos combinados (15); explicação do uso/ação dos produtos (13); atendimento pós venda (14); gentileza no atendimento (2); limpeza e higiene (5); organização da empresa (4); condições de pagamento (17); diversidade dos produtos (9) e agilidade na entrega (11).

Já no Quadrante II da figura 4 observam-se cinco atributos, considerados os pontos fracos da pesquisa: agilidade no recebimento (12); preço dos produtos (18); descontos para pagamento à vista (16); disponibilidade de estoque (10) e prontidão no atendimento (3).
No Quadrante III observam-se três atributos, considerados os pontos fracos menores da pesquisa: apresentação dos funcionários (1); aparência interna (7) e aparência externa (6). E, no Quadrante IV da figura 4 não há relação de atributos.

\section{Modelo Kano}

A análise de dados segundo o modelo Kano encontra-se representada na figura 5 através do gráfico de dispersão do índice de satisfação do cliente. Neste caso, a linha divisória dos quadrantes foi estabelecida em $+1,00$ para a escala do CS (coeficiente de satisfação) e $-1,00$ para o CI (coeficiente de insatisfação).

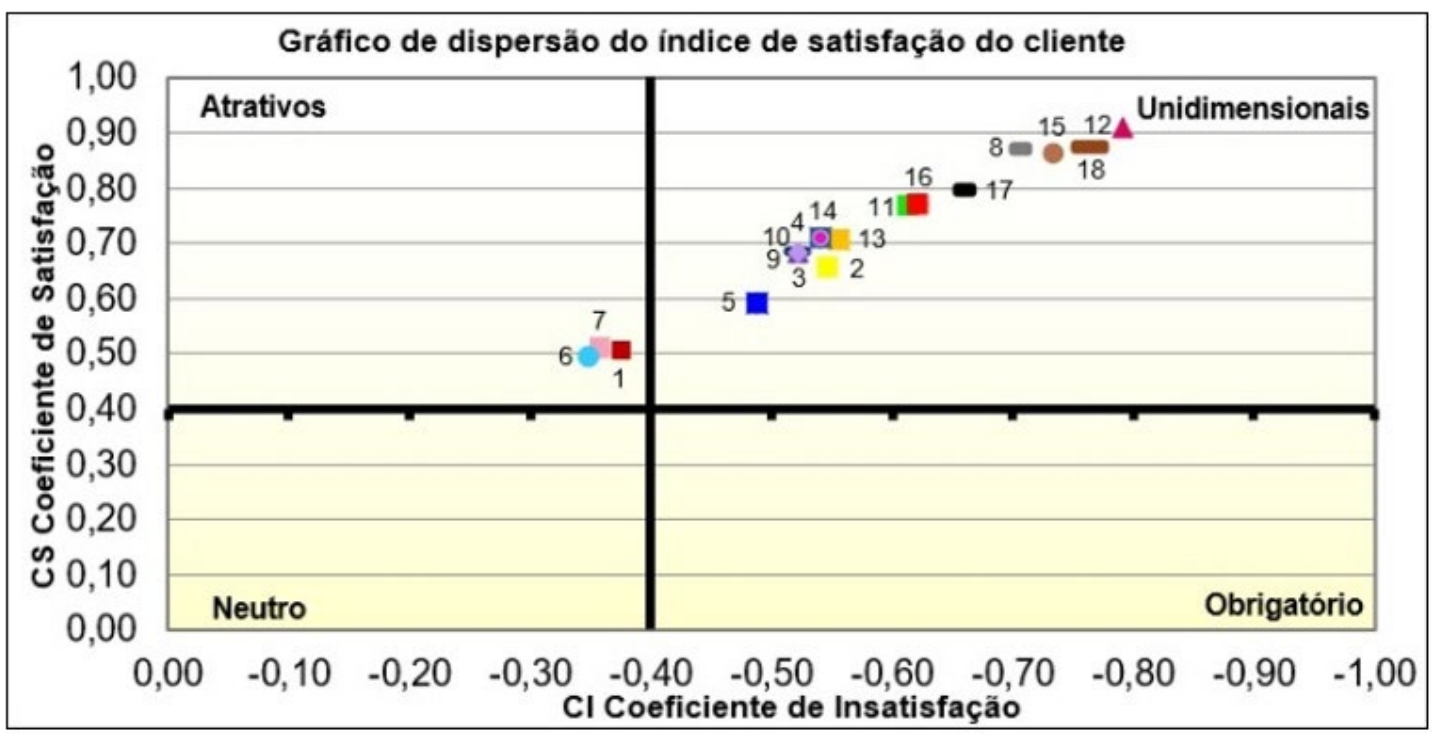

Figura 5. A Gráfico de dispersão do índice de satisfação do cliente. Fonte: elaboração própria pelos autores. 
A figura 5 pode ser visualizada de forma ampliada (zoom) por meio da figura 6.

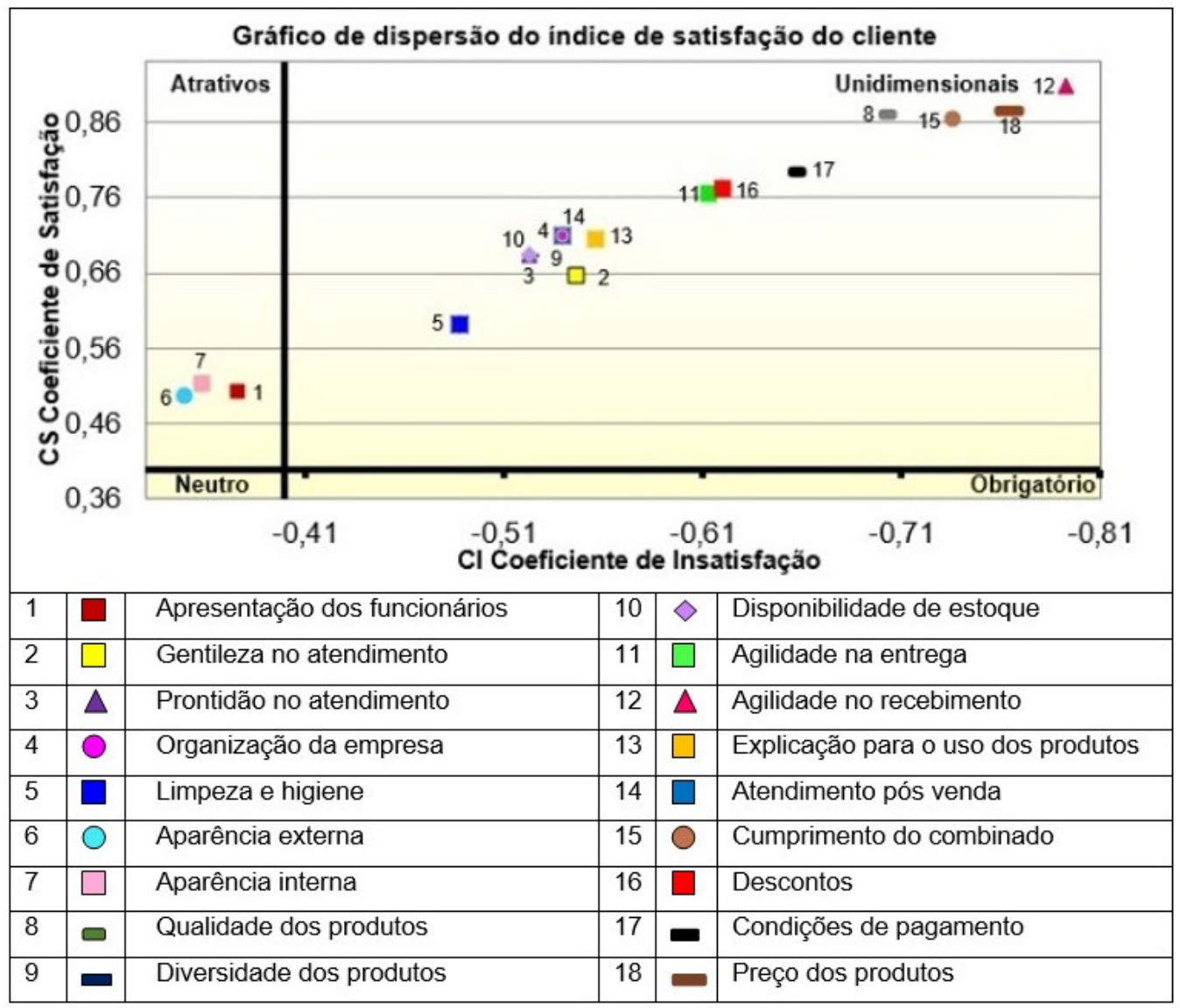

Figura 6. Ampliação da visualização do gráfico de dispersão do índice de satisfação do cliente. Fonte: elaboração própria pelos autores.

Por meio da figura 6, identifica-se que os 18 atributos relacionados encontram-se divididos entre atributos atrativos e unidimensionais. Os atributos atrativos apresentam maior impacto na satisfação se melhorados, são eles: aparência externa (6); aparência interna (7) e apresentação dos funcionários. Já os atributos unidimensionais se caracterizam por apresentarem impacto na satisfação proporcional ao nível de desempenho, são eles: agilidade no recebimento (12); preço dos produtos (18); cumprimento do combinado (15); qualidade dos produtos (8); condições de pagamento (17); descontos (16); agilidade na entrega (11); explicação para o uso dos produtos (13); atendimento pós-venda (14); organização da empresa (4); gentileza no atendimento (2); diversidade dos produtos (9); prontidão no atendimento (3); disponibilidade de estoque (10) e limpeza e higiene (5).

Observa-se que não houve incidência nos quadrantes de atributos neutros e obrigatórios. 


\section{Decisões de melhoria: Análise conjunta da Matriz de Importância $X$ Desempenho e modelo Kano}

0 modelo Kano introduz questionamentos sobre o uso da análise da Matriz de Importância $X$ Desempenho para a identificação de oportunidades de melhoria de produtos e serviços. A importância de um atributo varia conforme sua classificação pelo modelo Kano.

O tabela 10 mostra alguns problemas e decisões errôneas que podem ser tomadas quando não considerada a classificação dos atributos dentro do modelo Kano.

Tabela 10. Modelo Kano x erros na análise de Importância X Desempenho

\begin{tabular}{|c|c|c|c|c|c|}
\hline Modelo Kano & Importância & $\begin{array}{c}\text { Desempenho } \\
\text { competitivo }\end{array}$ & Decisão tomada & Razões do erro & Resultado \\
\hline Obrigatório & Alta & Igual & Melhorar & $\begin{array}{l}\text { Melhoria de } \\
\text { atributos } \\
\text { obrigatório } \\
\text { acima deste nível } \\
\text { não aumenta a } \\
\text { satisfação }\end{array}$ & $\begin{array}{l}\text { Desperdício de } \\
\text { recursos. }\end{array}$ \\
\hline Obrigatório & Baixa & Superior & Abandonar & $\begin{array}{l}\text { Experiências } \\
\text { anteriores } \\
\text { gratificantes e } \\
\text { desempenho } \\
\text { superior levam } \\
\text { o consumidor a } \\
\text { classificar atributo }\end{array}$ & $\begin{array}{l}\text { Desatenção a um } \\
\text { nível de serviço } \\
\text { necessário, } \\
\text { podendo levar à } \\
\text { insatisfação }\end{array}$ \\
\hline Obrigatório & Alta & Inferior & Melhorar & - & Decisão corretora \\
\hline Atrativo & Baixa & Inferior/igual & Abandonar & $\begin{array}{l}\text { onsumidores } \\
\text { sem experiência } \\
\text { anterior }\end{array}$ & $\begin{array}{l}\text { Oportunidade } \\
\text { de melhoria } \\
\text { relevante, pode ser } \\
\text { ignorada }\end{array}$ \\
\hline Atrativo & Alta & Igual & Melhorar & $\begin{array}{l}\text { Experiência } \\
\text { anterior com } \\
\text { outro serviço, } \\
\text { leva o consumidor } \\
\text { a considerar o } \\
\text { atributo importante }\end{array}$ & $\begin{array}{l}\text { Melhoria de um } \\
\text { atributo não- } \\
\text { crítico. }\end{array}$ \\
\hline Atrativo & Alta & Superior & Manter & - & Decisão Correta \\
\hline Unidimensional & Alta & $\begin{array}{l}\text { Inferior (Ponto } \\
\text { Fraco) }\end{array}$ & Melhorar & - & Decisão Correta \\
\hline Unidimensional & Alta & $\begin{array}{l}\text { Superior } \\
\text { (Ponto Forte) }\end{array}$ & $\begin{array}{l}\text { Manter ou } \\
\text { Melhorar em } \\
\text { segundo plano }\end{array}$ & - & Decisão Correta \\
\hline
\end{tabular}

Fonte: Tontini et al. (2004, p. 8-9). 
De modo geral, entende-se que o consumidor tende a dar maior importância para atributos obrigatórios, decrescendo essa importância para atributos unidimensionais, atrativos e neutros respectivamente. Como um desempenho superior em atributos obrigatórios não causa satisfação superior, a análise da Importância X Desempenho pode levar uma empresa a concentrar esforços em atributos cuja melhoria não trará grandes incrementos na satisfação geral. Já a baixa importância dada a atributos atrativos pode levar uma empresa a não melhorar atributos que poderiam trazer um diferencial em relação à concorrência, pois os mesmos geram uma satisfação superior, tratando-se de uma ação não esperada pelos consumidores, como por exemplo, a apresentação dos funcionários.

Analisando-se por conjunção a Matriz de Importância X Desempenho (figura 3) e o modelo Kano (figura 5), no Quadrante II da figura 1 observam-se cinco atributos, considerados os pontos fracos da pesquisa, conforme informado na tabela pelo item: agilidade no recebimento de produtos (12); preço dos produtos (18); descontos para o pagamento a vista (16); disponibilidade de estoque (10) e prontidão no atendimento (3); os quais deverão receber atenção imediata para melhoria, já que pela análise da figura 5, os atributos são considerados unidimensionais, ou seja, quanto maior o seu nível de desempenho, mais será a satisfação do cliente, e vice-versa.

Concluindo a análise baseando-se por meio de ambas ferramentas, verificou-se que a empresa tem a oportunidade de avaliar e buscar implementação de melhoria nos atributos descritos acima, que foram os considerados de baixa importância a ser melhorados. Nada impede a empresa de, futuramente, analisar todo o conjunto de atributos, já que estes foram considerados unidimensionais, baseando-se nestes para atingir um maior nível de desempenho garantindo elevação no nível de satisfação geral.

\section{Análise de correlação no SPSS}

Outro método empregado para análise de satisfação é a análise de correlação por meio do software SPPS versão 22, gerando a análise encontrada na tabela 11.

O coeficiente de correlação de Spearman (1904) é uma medida de associação que exige que ambas as variáveis se apresentem em escala de mensuração pelo menos ordinal, de modo que os elementos em estudo possam dispor-se por postos em duas séries ordenadas. Este teste nãoparamétrico destina-se a determinar o grau de associação entre duas variáveis X e Y, dispostas em pontos ordenados, o objetivo é estudar a correlação entre duas classificações (Bauer, 2007). 
Tabela 11. Correlação de Spearman x média da satisfação

\begin{tabular}{|c|c|c|c|c|}
\hline Atributo & Correlação & Significância & Satisfação média & Ranking \\
\hline \multicolumn{5}{|l|}{ Abaixo da média } \\
\hline Agilidade no rec. dos produtos (12) &, $548 * \star$ & Substancial & 2,18 & 1 \\
\hline Preço dos produtos (18) &, $580^{\star \star}$ & Substancial & 2,4 & 2 \\
\hline Aparência dos funcionários (1) &, $596 * \star$ & Substancial & 2,4 & 3 \\
\hline Descontos no pag. a vista (16) &, $501^{\star \star}$ & Substancial & 2,54 & 4 \\
\hline Disponibilidade de estoque (10) &, $555^{\star \star}$ & Substancial & 2,56 & 5 \\
\hline Aparência Interna (7) &, $677^{\star \star}$ & Substancial & 2,86 & 6 \\
\hline Prontidão no atendimento (3) &, $666^{\star \star}$ & Substancial & 2,9 & 7 \\
\hline Aparência externa (6) &, $657^{\star \star}$ & Substancial & 2,93 & 8 \\
\hline \multicolumn{5}{|l|}{ Acima da média } \\
\hline Qualidade dos produtos (8) &, $564^{\star \star}$ & Substancial & 3,8 & A \\
\hline Cumprimento com o combinado (15) &, $620^{\star *}$ & Substancial & 3,55 & B \\
\hline Explicação uso/ação produtos (13) &, $536^{\star \star}$ & Substancial & 3,35 & $\mathrm{C}$ \\
\hline Limpeza e higiene (5) &, $590^{\star \star}$ & Substancial & 3,3 & D \\
\hline Gentileza no atendimento (2) &, $672^{\star \star}$ & Substancial & 3,29 & $\mathrm{E}$ \\
\hline Atendimento pós venda (14) &, $670^{\star \star}$ & Substancial & 3,22 & $\mathrm{~F}$ \\
\hline Organização da empresa (4) &, $670^{\star \star}$ & Substancial & 3,17 & G \\
\hline Condições de pagamento (17) &, $617^{\star \star}$ & Substancial & 3,06 & $\mathrm{H}$ \\
\hline Diversidade dos produtos (9) &, $539^{\star \star}$ & Substancial & 3,03 & 1 \\
\hline Agilidade entrega dos produtos (11) &, $536^{\star *}$ & Substancial & 2,96 & $J$ \\
\hline Satisfação geral (19) & 1,000 & & 2,95 & \\
\hline
\end{tabular}

Fonte: elaboração própria pelos autores.

A classificação de correlação de Spearman $\mathrm{x}$ média de satisfação é classificada em: 0,0 até $0,20^{*}$ correlações nulas; 0,21 até $0,40^{*}$ correlações fracas; 0,41 até $0,70^{*}$ correlações substanciais; 0,71 até $0,90^{*}$ correlações fortes; 0,91 até 1,0 correlações extremamente fortes (Rodrigues, 2010).

De acordo com os dados analisados, observase que 10 desses tópicos apresentam uma satisfação média maior que a satisfação geral (tabela 11), sendo assim, esses tópicos são de extrema importância para os clientes, porém, estão satisfazendo os mesmos, dessa forma dispensam uma atenção imediata para a melhoria. Já os 8 tópicos que são menores que a satisfação geral e são marcados como importantes para os clientes, são: agilidade no recebimento dos produtos (12); preço dos produtos (2); apresentação dos funcionários (1); descontos no pagamento a vista (16); disponibilidade de estoque (10); aparência interna (7); prontidão no atendimento (3) e aparência externa (6); estes devem ser analisados minuciosamente para melhoria, pois, são de extrema importância para os clientes e estão abaixo da média relacionada.

Todos os tópicos devem ser avaliados pela empresa, pois, apesar de grande parte dos tópicos passarem da satisfação média da empresa, a mesma tem um índice baixo e que pode ser explorado para melhor atrair clientes novos e melhorar a satisfação dos atuais clientes, fidelizando os mesmos. 


\section{Pontos fortes, pontos fracos $e$ sugestões de melhoria}

Solicitou-se aos respondentes que indicassem de forma descritiva, pontos fortes em relação aos produtos e serviços prestados pela empresa do setor agrícola. Dos 100 respondentes, obtiveramse 100 contribuições para a resposta. Os dados obtidos são apresentados na tabela 12:

Tabela 12. Pontos fortes indicados

\begin{tabular}{lc|}
\hline Pontos fortes & Citações \\
\hline Localização da empresa & 28 \\
\hline Boa relação com funcionários & 24 \\
\hline Agilidade no atendimento & 19 \\
\hline Produtos de qualidade & 13 \\
\hline Pagamento à vista & 10 \\
\hline Honestidade & 6 \\
\hline
\end{tabular}

Fonte: elaboração própria pelos autores.

Percebe-se uma grande satisfação dos clientes no que diz respeito à localização da empresa, a boa relação com os funcionários e a agilidade no atendimento.

Outros pontos fortes também se apresentam relevantes, como a qualidade dos produtos, pagamento à vista e a honestidade da equipe para com os clientes.

Dos 100 respondentes, cerca de 77 responderam à questão sobre os pontos fracos. Os dados obtidos são apresentados na tabela 13:

Tabela 13. Pontos fracos indicados

\begin{tabular}{lc}
\hline Pontos fracos & Citações \\
\hline $\begin{array}{l}\text { Muitos descontos no recebimento dos } \\
\text { produtos }\end{array}$ & 25 \\
\hline Falta agilidade no recebimento de cargas & 13 \\
\hline Pouco espaço para armazenamento de cereais & 12 \\
\hline Espaço pequeno para manobras de caminhões & 10 \\
\hline A empresa não possui secador de cereais & 5 \\
\hline $\begin{array}{l}\text { Pagamento adicional inexistente na venda } \\
\text { imediata }\end{array}$ & 5 \\
\hline Entrega produtos ao cliente & 4 \\
\hline Desigualdade entre clientes & 3 \\
\hline
\end{tabular}

Fonte: elaboração própria pelos autores.
É possível verificar por meio da tabela 13, que os pontos fracos com maior percepção por meio dos clientes são: muitos descontos no recebimento dos produtos; falta agilidade no recebimento de cargas; pouco espaço para armazenamento de cereais e espaço pequeno para manobras de caminhões. Outros pontos fracos também se apresentam significativos: falta de secador de cereais; pagamento adicional inexistente na venda imediata; entrega de produtos aos clientes e desigualdade entre clientes.

Em relação aos problemas citados pelos clientes é possível realizar uma análise e desenvolver estratégias para solucioná-las. Conforme defende Berry (1995), a empresa deve identificar todas as decepções do cliente, mesmo que pequenas, pois compreendendo o comportamento do consumidor, torna-se possível reparar as frustrações e conseguir mantê-los na empresa.

Dos 100 respondentes, obtiveram-se 92 respostas à questão de sugestões de melhoria. Os dados obtidos são apresentados na tabela 14 .

Tabela 14. Sugestões de melhoria

\begin{tabular}{lc|}
\hline Sugestões & Citações \\
\hline $\begin{array}{l}\text { Aumentar a capacidade de recebimento de } \\
\text { grãos }\end{array}$ & 23 \\
\hline Melhorar o acesso à firma & 19 \\
\hline Ampliar o estacionamento para caminhões & 14 \\
\hline Melhorar recebimento de grãos & 13 \\
\hline $\begin{array}{l}\text { Diminuir os descontos no recebimento de } \\
\text { produtos }\end{array}$ & 13 \\
\hline $\begin{array}{l}\text { Igualdade entre os clientes } \\
\text { Aumentar a entrega de produtos ao cliente }\end{array}$ & 7 \\
\hline \multicolumn{2}{c}{ Fonte: elaboração própria pelos autores. } \\
\hline
\end{tabular}

Observa-se, por meio da tabela 14, que os clientes almejam algumas mudanças nos serviços prestados pela empresa. As sugestões que se evidenciam mais relevantes são: aumentar a capacidade de recebimento de grãos; melhorar o acesso à firma; ampliar o estacionamento para caminhões; melhorar e diminuir os descontos no recebimento de grãos. 


\section{Considerações finais}

Definiu-se como objetivo da pesquisa a análise de oportunidades de melhoria na satisfação de cliente da empresa Agrícola Horizonte, pelo uso conjunto de duas ferramentas: Matriz de Importância X Desempenho e o modelo Kano. Os resultados encontrados demonstram a eficácia dos métodos de análise empregados para mensuração, contribuindo na melhoria das decisões a serem tomadas.

Empregou-se, nesse estudo, a apuração do grau de satisfação dos clientes de acordo com cada atributo pesquisado, correlacionando os dados e analisando-se os resultados encontrados. No questionário, o respondente indicou o seu grau de satisfação em escala de (5) extremamente satisfeito e (-5) extremamente insatisfeito, podendo afirmar, ainda, como se sentiria se um atributo melhorasse ou piorasse.

O objetivo da pesquisa foi concluído com sucesso, atingindo a quantidade mínima de respondentes (100 questionários). Na análise da satisfação média, obteve-se o índice de 81,36 $\%$ no quesito se o cliente voltaria ou indicaria os produtos e serviços da empresa. Esse percentual indica que a empresa cumpre com os requisitos necessários para deixar o cliente satisfeito com a mesma. Porém, verificou-se que alguns atributos precisam ser analisados e melhorados para que haja uma maior satisfação dos clientes, pra quem sabe, futuramente, atingir um grau de satisfação ainda maior.

Todos os itens pesquisados apresentaram correlações substanciais, sendo que oito deles obtiveram uma nota inferior a satisfação média. Nessa análise percebeu-se que a empresa precisa focar nos tópicos que foram avaliados como importantes e não atingiram a média final, para manter seus clientes e conquistar novos, e, que assim possa continuar crescendo e maximizando seu percentual de lucro. 


\section{Referências}

Anderson, E., Fornell, C. and Rust, R. (1997). Customer satisfaction, productivity, and profitability: Differences between goods and services. Marketing Science, 16(2), 129-145.

Anderson, E. (1998). Customer satisfaction and wordof-mouth. Journal of Service Research, 1(1), 5-17.

Anderson, E. and Fornell, C. (1999). The customer satisfaction index as a leading indicator. Em Iacobucci, D. and Swartz, T. (Ed.), Handbook of services marketing and management (pp. 255-267). New York, USA: SAGE Publications.

Babin, B. and Harris, W. (2012). Consumer Behavior. Mason, USA: Cengage Learning.

Bateson, J. e Hoffman, K. (2001). Marketing de serviços. Porto Alegre, Brasil: Bookman Editora.

Bauer, L. (2007). Estimação do Coeficiente de Correlação de Spearman Ponderado (tese de pos-graduação). Faculdade de Medicina, Universidade Federal do Rio Grande do Sul, Porto Alegre, Brasil.

Berry, L. (1995). Relationship marketing of servicesGrowing interest, emerging perspectives. Journal of the Academy of Marketing Science, 23(4), 236-245.

Candeloro, R. e Almeida, S. (2002). Correndo pro abraço: como vender mais, fazendo com que o cliente compre sempre. Salvador, Brasil: Casa da Qualidade.

Kano, N. et al. (1984). Attractive quality and must-be quality. Journal of the Japanese Society for Quality Control, 14(2), 39-48.

Kano, N. (1991). A qualidade atrativa e a obrigatória. Tokyo, Japan: AOTS.

Kotler, P. e Armstrong, G. (1999). Princípios de marketing . Rio de Janeiro, Brasil: LTC Editora.

Kotler, P. (2000). Administração de marketing: a edição do novo milênio. São Paulo, Brasil: Prentice Hall.

Kotler, P. e Keller, K. (2006). Administração de Marketing. São Paulo, Brasil: Pearson \& Prentice-Hall.

Martilla, J.A. and James, J.C. (1977). ImportancePerformance Analysis. Journal of Marketing, 41(1), 77-79.

Mattar, F.N. (1996). Pesquisa de marketing. São Paulo, Brasil: Atlas.
Matzler, K. et al. (2004). The asymmetric relationship between attribute-level performance and overall customer satisfaction: A reconsideration of the importance-performance analysis. Industrial Marketing Management, 33(4), 271-277.

Milan, G.S. e Trez, G. (2005). Pesquisa de satisfação: um modelo para planos de saúde. RAE-eletrônica, 4(2). Recuperado de http://www.scielo.br/pdf/raeel/ v4n2/v4n2a02.pdf.

Pereira, R., Tontini, G. e Silveira, A. (2005). Funções, atividades e responsabilidades da administração de compras como parte da logística empresarial: comparação entre empresas catarinenses e norteamericanas. Revista de Negócios, 10(3), 217-229.

Rodrigues, W.C. (2010). Estatística aplicada. Paracambi, Brasil: IST.

Salazar, G.T. (2001). Administração geral: teoria e gerência das organizações. Lavras, Brasil: Universidade Federal de Lavras.

Solomon, M. (1999). Consumer Behavior: Buying, having and being. New Jersey, USA: Prentice Hall.

Spearman, C. (1904). "General intelligence," objectively determined and measured. The American Journal of Psychology, 15(2), 201-292.

Tontini, G. (2003). Como identificar atributos atrativos e obrigatórios para o consumidor. Revista de Negócios, 8(1), 19-28.

Tontini, G. and Silveira, E. (2005). Identification of critical attributes of success in products and services: An alternative to importance - performance analysis. Em BALAS Annual Conference Proceedings, Instituto de Empresa, Madrid, España.

Tontini, G. and Silveira, A. (2007). Identification of satisfaction attributes using competitive analysis of the improvement gap. International Journal of Operations \& Production Management, 27(5), 482500.

Tontini, G. et al. (2019). Análise de oportunidades de melhoria em laboratórios fotográficos através da integração da matriz de importância $\mathrm{x}$ desempenho com o modelo Kano de qualidade. Revista de Negócios, 9(3), 274-294. 
Zeelenberg, M. and Pieters, R. (2004). Beyond valence in customers' dissatisfaction: A review and new findings on behavioral response to regret and disappointment in failed services. Journal of Business Research, 57(4), 445-455. 